\title{
REVISIÓN HERMENÉUTICA DE LA TRADICIÓN INTERNALISTA EN FILOSOFÍA DEL DEPORTE
}

\section{A HERMENEUTICAL REVIEW OF THE INTERNALIST TRADITION IN THE PHILOSOPHY OF SPORT}

\author{
Francisco Javier López y Xavier Gimeno ${ }^{1}$ \\ Pennsylvania State University y Universidad de Valencia
}

Recibido: 2-9-2015

Aceptado: 12-3-2016

Resumen: En este trabajo proponemos un análisis hermenéutico de la tradición dominante en filosofía del deporte: el internalismo. Para ello, identificamos los prejuicios que dirigen su concepción del deporte, a saber: el prejuicio platónico-analítico. Tras ello, ponemos en cuestión cuatro consecuencias problemáticas de seguir este prejuicio: (a) sesga la realidad sin que lo percibamos; (b) dulcifica la realidad del deporte al apelar únicamente a la idea de excelencia física; (c) la idea de excelencia física en que se basa es problemática; y (d) nos hace dar un salto cuanto menos dudoso e injustificado de la descripción del fenómeno deportivo a los requisitos normativos referidos a cómo debe practicarse el deporte.

Palabras clave: hermenéutica, filosofía del deporte, prejuicios, Heidegger, internalismo deportivo, excelencia.

\begin{abstract}
In this paper, we make a hermeneutical analysis of the prevalent tradition in the philosophy of sport, that of internalism. To do so, we identify the prejudices that guide the internalist conception of sport, namely: the Platonic-Analytic prejudice introduced by Suits, who is one of the forefathers of the internalist approach. Then, we critically analyze four consequences of following such a prejudice: (a) unknowingly reduces reality; (b) generates a sugar-coated view of sport; (c) the idea of physical excellence is fuzzy; and (d) misguided because it generally leads to an unjustified and problematic leap from the descriptive analysis of the sporting phenomenon to the setting of normative requirements about how to practice sport.
\end{abstract}

Keywords: hermeneutics, philosophy of sport, prejudices, Heidegger, internalism, excellence.

1. (francisco.javier.lopez@uv.es) Franciso Javier López, Pennsylvania State University Kinesiology Department y Rock Ethics Institute (Estados Unidos). Xavier Gimeno, Universidad de Valencia (España), Facultad de Filosofía y CC.EE, Departamento de filosofía del derecho, moral y política. 


\section{El objetivo de una teoría hermenéutica del deporte}

La filosofía del deporte, al haber surgido en el ámbito filosófico anglo-americano, ha estado desde sus comienzos claramente dominada por una metodología y objetivos claramente analítico-lingüísticos. ${ }^{2}$ No obstante, los análisis de corte filosófico más continentales han ido poco a poco apareciendo en esta disciplina, impregnándola con un carácter más descriptivo-narrativo, así como de sus objetivos filosóficos propios. ${ }^{3}$ La diferencia esencial, en este sentido, entre filosofía anglo-americana y continental es que esta última está menos relacionada con la búsqueda lingüística de definiciones y conceptos, y más centrada en la clarificación crítica e iluminación de un tema. Una propuesta continental en auge desde principios del siglo xx es la hermenéutica. La cual nos proponemos utilizar para: (a) comprender mejor el fenómeno deportivo y; (b) complementar la filosofía del deporte.

Siguiendo a Shaun Gallagher, el objetivo de la hermenéutica es el de desvelar todos aquellos "factores epistemológicos, sociológicos, culturales, y lingüísticos" ${ }^{4}$ que influyen en nuestra comprensión de la realidad. Con el fin de comprender mejor el fenómeno deportivo, nosotros queremos desvelar algunos de los factores determinantes de nuestra concepción filosófica del deporte. Para ello, realizaremos una interpretación hermenéutico-crítica de la propuesta dominante en la filosofía del deporte, a saber: el internalismo amplio o interpretacionismo, cuyos mayores defensores son Robert L. Simon, César R. Torres, y John S. Russell, entre otros. ${ }^{5}$

Siendo la propuesta dominante en nuestra teorización filosófica sobre el deporte, el internalismo tiene una importante influencia sobre nuestras interpretaciones y preconcepciones intersubjetivas de esta actividad social. Así, por ejemplo, la caracterización internalista del deporte como práctica pedagógica ha sido aceptada sobremanera por instituciones deportivas tan relevantes como el Comité Olímpico Internacional (COI) o

2. McNamee, M.:"Sport, ethics and philosophy; context, history, prospects." En Sport, Ethics and Philosophy 1(1), 2007, pp. 1-6; Kretchmar, R. S.: «The philosophy of sport and analytic philosophy" en C. R. Torres, Bloomsbury companion to the philosophy of sport, London: Bloomsbury, 2014, pp. 41-52.

3. Moe, V. F. :《The philosophy of sport and continental philosophy» en C.R. Torres, Bloomsbury companion to the philosophy of sport, London: Bloomsbury, 2014, pp. 52-66; Aggerholm, K.: Talent development, existential philosophy and sport: on becoming an elite athlete, New York, Routledge, 2015.

4. Gallagher, S.: Hermeneutics and education, Albany: State University of New York Press, 1992 p. 5

5. Russell, J. S.: "Are rules all an umpire has to work with?" en Journal of the Philosophy of Sport 26(1), 1992, pp. 27-49; Russell, J. S.:"Moral realism in sport." En Journal of the Philosophy of Sport 31(2), 2004,pp. 142-160; Simon, R. L.: «Theories of sport» en C.R. Torres, The Bloomsbury companion to the philosophy of sport, cit., pp. 93-98; Torres, C. R.:"Furthering interpretivism's integrity: Bringing together ethics and aesthetics." enJournal of the Philosophy of Sport39(2), 2012, pp. 299-319.

Thémata. Revista de Filosofía No54 (2016) pp.: 125-148. 
la Federación Internacional de Fútbol Asociación (FIFA). Por ello, resulta relevante analizar la propuesta internalista con cuidado. Según ésta, como ya hemos adelantado, el deporte es:

Una búsqueda conjunta de la excelencia, una actividad que es cooperativa de modo significativo en el sentido de que los participantes consienten ser puestos a prueba a través del rasero de la competición por el valor intrínseco que posee superar desafíos interesantes, aprendiendo sobre nosotros y otros a través del intento de superar pruebas competitivas. ${ }^{6}$

Es más, existe una afinidad íntima entre el internalismo amplio en filosofía del deporte y la hermenéutica. ${ }^{7}$ Por ejemplo, el concepto de interpretación es clave para ambos. Este concepto se refiere al modo de comprender la realidad que enfatiza nuestra ligazón con todo aquello que nos influye constantemente, como, por ejemplo, el pasado y el futuro, o nuestra sociedad y circunstancias. No obstante, la interpretación no es un modo cualquiera de interpretar la realidad, sino el más propiamente humano. Como muestra Nietzsche ${ }^{8}$, los seres humanos nos encontramos siempre forzados a interpretar la realidad con el fin de darle respuesta. Somos un "animal fantástico" 9 que vive de interpretaciones. ${ }^{10}$

Dado que las interpretaciones no son subjetivas, sino compartidas y heredadas de la tradición de la que formamos parte, la hermenéutica nos sitúa en un plano intersubjetivo. Por ejemplo, las primeras "verdades" que aprendemos no las descubrimos de modo individual, sino que las heredamos de la tradición en la que estamos inscritos cultural y socialmente. Igualmente, nuestra comprensión de la realidad siempre está mediada por elementos intersubjetivos como, por ejemplo, el lenguaje, los prejuicios, o las ideas comúnmente aceptadas. Por esto, algunos autores afirman que el presupuesto básico de la hermenéutica es: "todo lo que hay es interpretación".

En filosofía del deporte, los autores que más han enfatizado este carácter intersubjetivo de nuestras concepciones del deporte son William

\section{Simon, R. "Theories of Sport" cit., p. 47.}

7. López Frías, F. J.: "Broad internalism and interpretation. The plurality of interpretivist approaches.” En S. Klein, Defining sport. Contemporary explorations, 2015, Lexintong Books. En prensa.

8. Aunque Nietzsche no se concibe a sí mismo como un autor hermenéutico, sí que ha habido ciertos estudiosos que le han concebido como uno de los padres de esta propuesta filosófica, debido a su filosofía perspectivista. «En todo caso hablar del espíritu y del bien como lo hizo Platón significaría poner la verdad cabeza abajo y negar el perspectivismo, el cual es condición fundamental de toda vida.» Nietzsche, F.: Más allá del bien y del mal, Madrid: Alianza, 2013, p. 25.

9. Conill-Sancho, J.: "La superación del naturalismo en Ortega y Gasset." En Isegoría 46, 2012, pp. 167-192.

10.«El hombre se ha ido convirtiendo paulatinamente en un animal fantástico cuya existencia ha de cumplir una condición más que en otro animal: de cuando en cuando, el hombre tiene que creer saber por qué existe». Nietzsche, F.:La gaya ciencia, Madrid: EDAF, 2002, p. 69.

Thémata. Revista de Filosofía $\mathrm{N}^{\circ} 54$ (2016) pp.: 125-148. 
J. Morgan, César R. Torres y Douglas W. McLaughlin. ${ }^{11}$ No obstante, no puede decirse que estos autores lleven a cabo análisis propiamente hermenéuticos del deporte, sino que, como mucho, tratan de fundamentan algunas de sus propuestas en ideas de autores influidos por la hermenéutica como: Alasdair MacIntyre, Richard Rorty, John Rawls, o Andrei Marmor. ${ }^{12}$

Los análisis propiamente hermenéuticos son escasos en la filosofía del deporte. Entre ellos, hemos de destacar los de Andrew Edgar y Kenneth Aggerholm. ${ }^{13} \mathrm{El}$ primero ha basado su propuesta en la hermenéutica de Gadamer y Habermas, mientras que el segundo, partiendo de la fenomenología de Sartre y de Merleau-Ponty, complementa su propuesta fenomenológica con otros autores más propiamente hermenéuticos como Gadamer o Sloterdijk.

Por el contrario, los trabajos basados en la fenomenología sí son más comunes en la filosofía del deporte, como por ejemplo los de Arno Müller, Jim Parry e Irena Martinkova, Ivo Jirasek, Gunnar Breivik, y R. Scott Kretchmar. ${ }^{14}$ Todos estos autores ofrecen un análisis fenomenológico del deporte basado en propuestas como las de Heidegger, Merleau Ponty, y Husserl. Esta combinación de fenomenología y hermenéutica no es casual, pues ambas corrientes filosóficas no sólo surgieron históricamente como parte de un continuo, sino que suelen ser confundidas y equiparadas habitualmente dentro de la filosofía del deporte. Sin embargo, este es un error que trataremos de evitar aquí.

Para la fenomenología, cuya presencia es mayor dentro de la filosofía del deporte actual, el objetivo principal es el de describir el modo en el que los fenómenos constitutivos de la práctica deportiva se presentan en nuestra conciencia y, así, tratar de ir "a las cosas mismas"15. En un

11. McLaughlin, D. W. y Torres C.R.: "Sweet tension and its phenomenological description: Sport, intersubjectivity and horizon." En Sport, Ethics and Philosophy Sport, Ethics and Philosophy 5(3), 2011, pp. 270-284; Morgan, W. J.: "Broad internalism, deep conventions, moral entrepreneurs, and sport." en Journal of the Philosophy of Sport 39(1), 2012,pp. 65-100.

12. MacIntyre, A: Tras la virtud, Barcelona: Crítica, 2001; Marmor, A.: Social conventions: from language to law, Princeton, N.J: Princeton University Press, 2009; Rawls, J.: Liberalismo político, México: Fondo de Cultura Económica, 1995; Rorty, R.: Philosophy and the mirror of nature, Princeton: Princeton University Press, 1979.

13.Aggerholm, op. cit; Edgar, A.:Sport and art: an essay in the hermeneutics of sport, London: New York, Routledge, 2014.

14. Kretchmar, R. S.: "From test to contest: An analysis of two kinds of counterpoint in sport." En Journal of the Philosophy of Sport 2(1), 1975, pp. 23-30; Martinkova, I. y Parry J.: Phenomenological approaches to sport, New York: Routledge, 2012.

15. Para Husserl, padre de la fenomenología, y sus seguidores este método es el de la epojé. El cual nos permite suspender nuestra "actitud natural" respecto al mundo, es decir, nuestra situación contextual, para captar los fenómenos en sí mismos, tal y como se nos aparecen a nuestra consciencia.

Thémata. Revista de Filosofía No54 (2016) pp.: 125-148. 
trabajo de fenomenología clásico en filosofía del deporte, R. Scott Kretchmar aplica la epojé -غ̇похn்-, es decir, la suspensión del juicio o actitud "natural" respecto al mundo y los fenómenos que lo constituyen. ${ }^{16}$ De ese modo, Kretchmar trata de realizar una reducción fenomenológica para captar los elementos definitorios del fenómeno deportivo a través de las estructuras intencionales envueltas en el practicar deporte (que, siguiendo a Merleu-Ponty, son esencialmente corporales). Para Kretchmar, los dos elementos definitorios del fenómeno del deporte son ser una prueba física (test) y ser un enfrentamiento con otro (contest).

En este sentido, la diferencia con el hermeneuta es más que clara. Dado su carácter descontextualizador, la epojé permite -en opinión del fenomenólogo clásico- encontrar aquellas estructuras epistemológicas universales y comunes a todos los seres que poseen conciencia y, a través de las cuales, el fenómeno se nos aparece en su estado puro, es decir, limpio de cualquier juicio de carácter inmediato y natural. El hermeneuta, por el contrario, apela a la vida fáctica de los sujetos, a aquello en lo que siempre estamos envueltos y no podemos poner en suspenso epistemológicamente hablando. Para Gadamer, la hermenéutica es la estructura del ser. ${ }^{17}$ Es decir, el ser se revela como interpretación. Dicha condición del ser entendida como interpretación se basa en la tradición y los prejuicios que la constituyen, así como en el carácter temporal, cambiante y dialéctico que los atraviesa. La hermenéutica ha de comprenderse, por lo tanto, como un sistema ontológico, es decir, referido en primera instancia a nuestro modo de ser y no tanto, como algunos creen erróneamente, a meras interpretaciones subjetivas. ${ }^{18}$

Así, por ejemplo, en Verdad y método, Gadamer afirma que cuando tratamos de comprender algo siempre proyectamos significado en la cosa a comprender. Para ello, recurrimos a una tradición que da sentido a aquello que carece de él. Los prejuicios se encargan de realizar esta proyección de sentido. Éstos nos presentan la realidad de un modo determinado y con una finalidad que nos vincula estrechamente a la tradición en la que esta-

16. Kretchmar, R.S, "From test to contest" cit.

17. Gadamer, H.G.: Verdad y método: fundamentos de una hermenéutica filosófica. Salamanca: Ediciones Sígueme, 1977.

18. Que todo ser sea interpretación e intérprete (de modo ontológico), no implica que todo sea contingente o subjetivo, sino que la interpretación hace referencia a aquellas estructuras que dotan de sentido a nuestra realidad, es decir, a una realidad histórica en que nos encontramos. Esta realidad está compuesta no sólo por el presente, sino por el pasado, en forma de tradición y prejuicios, y el porvenir, en forma de proyectos.

Thémata. Revista de Filosofía N54 (2016) pp.: 125-148. 
mos inmersos: «los prejuicios del individuo, mucho más que sus juicios, son la realidad histórica de su ser.» ${ }^{19}$

Siendo esto así, una teoría hermenéutica del deporte debe tomar como punto de partida el hecho de que existe una tradición formada por diversas concepciones del deporte. Esa tradición es la de las propuestas internalistas en filosofía del deporte, cuya historia ha ido dando lugar a diversas teorías del deporte. Todas ellas han acabado deviniendo en una teoría que hoy se adopta de modo mayoritario como la más adecuada dentro de la disciplina: el interpretacionismo o internalismo amplio. ${ }^{20}$ Así pues, nuestro primer fin será, pues, no sólo mostrar cuáles son los caracteres determinantes a la base de la concepción filosófica internalista del deporte dominante, sino, sobre todo, analizar la tradición internalista de la que ésta surge y recibe sus caracteres definitorios. Con ello, no tratamos de encontrar una propuesta internalista más adecuada que la actual, por ejemplo, a través de la captación de los elementos definitorios del deporte en general y del internalismo en particular. Más bien, trataremos de mostrar cuáles son los presupuestos y prejuicios que han atravesado y dirigido la tradición internalista del deporte que, a nuestro parecer, constituye el paradigma dominante en la actualidad. Con ello, localizaremos cuáles son los límites de la tradición filosófica predominante a la hora de estudiar el deporte.

\section{La génesis platónica de la propuesta internalista del deporte}

El padre intelectual del internalismo en filosofía del deporte es Bernard Suits, cuya propuesta filosófica, el internalismo formalista, es una respuesta a la posición anti-definicionalista wittgensteniana respecto al concepto de juego:

Considere, por ejemplo, los procedimientos que llamamos 'juegos'. Me refiero a juegos de mesa, de naipes, de pelota, juegos olímpicos y así sucesivamente. ¿Qué es lo común a todos ellos? —no diga: 'Debe haber algo en común, de lo contrario no serían llamados 'juegos', sino que mire y vea si hay cualquier cosa en común. Porque si usted los mira, no verá algo que es común a todos, sino más encima, semejanzas, relaciones y una serie entera de ellas. ${ }^{21}$

El hecho de responder al anti-definicionalismo wittgensteniano es una muestra de la naturaleza platónica de la propuesta suitsiana. Pues,

19. Ibidem, p. 175 .

20. LópezFrías, F. J.: La filosofía del deporte actual. Paradigmas y corrientes, Roma: (Qua. Pe.G) Università degli Studi di Roma, 2014.

21. Wittgenstein, L,Investigaciones filosóficas, § 66 .

Thémata. Revista de Filosofía №54 (2016) pp.: 125-148. 
con ello, Suits emula el fin principal de las filosofías de Sócrates y Platón: enfrentarse a la posición sofística anti-definicionalista. Así, por ejemplo, se atribuye al sofista Protágoras la idea de que, dado que el hombre es la medida de las cosas y la realidad es cambiante, no podemos encontrar una definición o esencia de las cosas, sino que éstas dependen del contexto:

Sóc.- Parece, ciertamente, que no has formulado una definición vulgar del saber, sino la que dio Protágoras. Pero él ha dicho lo mismo de otra manera, pues viene a decir que el hombre es medida de todas las cosas, tanto del ser de las que son, como del no ser de las que no son. ${ }^{22}$

Según la crítica de Protágoras a la búsqueda de definiciones platónica, dado que el punto de referencia para captar la verdad de las cosas es la naturaleza individual de cada uno, no pueden existir definiciones universales de las cosas. El propio Suits acepta en cierto modo esta idea, pues afirma que su propuesta no es platónica en el caso de todos los objetos a definir, sino sólo en el del concepto de juego. ${ }^{23}$ Aunque, a su juicio, puede haber realidades que carezcan de definición. No obstante, el juego y el deporte no son unas de ellas:

La orientación de este libro es filosófica en el sentido tradicional de la palabra. Está destinado a descubrir y formular una definición, y a seguir las implicaciones de tal descubrimiento incluso cuando ellas nos lleven a direcciones sorprendentes, e incluso a veces desconcertantes. ${ }^{24}$

En esta cita, podemos ver que la metodología filosófica empleada por Suits es claramente influida por el método dialéctico-platónico. Otro elemento que muestra claramente esta conexión entre la metodología platónica y la filosofía del deporte es que Suits construye su obra más famosa y en la que pretende aportar una definición de juego, The Grasshopper, como un diálogo al más puro estilo platónico. En él, la cigarra (en clara referencia a la fábula de Esopo) y sus dos discípulos Skepticus y Prudence entablan un diálogo con el fin de alcanzar una definición del juego.

Como hemos dicho, Suits procede según la metodología de la dialéctica platónica. Esto significa que establece hipótesis que han de ser probadas a través de la deducción racional para tratar de alcanzar una definición universal del juego. El objetivo de estas hipótesis es reducir la realidad del juego hasta percibir sus elementos definitorios que, en última instancia, serán los que compongan la Idea o Forma del mismo.

22. Platón, Teeteto, 152a.

23. Suits, B.:The grasshopper: games, life and utopia, Peterborough, Ont: Broadview Press, 2005.

24. Ibidem, p. ix.

Thémata. Revista de Filosofía $\mathrm{N}^{\circ} 54$ (2016) pp.: 125-148. 
Así, por ejemplo, afirma Suits que el diálogo con la cigarra sucede del siguiente modo:

Primero, él presentaba una definición de los juegos o, para ser incluso más preciso, una definición del jugar a juegos. Luego, me invitaba a someter esa definición a una serie de pruebas. Yo tenía que presentar contra la definición las objeciones más convincentes que se me ocurrieran, y él tenía que responder esas objeciones. ${ }^{25}$

En lo que sigue, trataremos de mostrar por qué, como heredero de la filosofía de Suits, cualquier otro tipo de internalismo no deja de ser un platonismo actualizado. Para cualquier modo de internalismo dentro del ámbito de la filosofía del deporte, el objetivo prioritario siempre pasa por tratar de alcanzar una definición esencial. Siendo éste el objetivo principal de la filosofía del deporte internalista, afirmamos que ésta se encuentra dominada por lo que denominaremos como "prejuicio platónico".

Es más, en otro nivel de discurso, encontramos otro ejemplo más de platonismo dentro de las diversas propuestas internalistas, a saber, el carácter definitorio común al que se apela para definir el deporte es el de la lucha por la excelencia. Si bien en la filosofía del deporte, en general, y en el internalismo deportivo, en particular, se ha superado el error platónico de separar categóricamente la mente del cuerpo. ${ }^{26}$ No obstante, hay un error platónico que se mantiene, a saber: la concepción que se ofrece de lo corporal, ligada a la excelencia física, acaba estando influida por la intención platónica de ligar nuestro análisis de la realidad a lo más excelso: lo ideal, lo supremo, lo brillante... Muestra clara de ello es que es el Sol el que en la alegoría de la Caverna simboliza tanto las ideas, como el objetivo a alcanzar por el filósofo, quien al final de su formación filosófica ha de ser capaz de mirar al Sol sin ser deslumbrado.

Concebir el deporte como lucha por la excelencia física ofrece una visión idealizada, tanto del deporte, como de nuestra naturaleza corporal. De este modo, la filosofía internalista queda ligada a un referente ideal e inalcanzable: la excelencia física. ${ }^{27}$ Por lo tanto, podríamos afirmar que el carácter platónico del internalismo acaba dando lugar a un "imperialismo

25. Ibidem, p. 17.

26. Kretchmar, R. S.: "Dualisms, dichotomies and dead ends: limitations of analytic thinking about sport." En Sport, Ethics and Philosophy 1(3), 2007, pp. 266-280.

27. Platón, República, L. 7.

Thémata. Revista de Filosofía №54 (2016) pp.: 125-148. 
de la excelencia" ${ }^{28}$ dentro de la filosofía del deporte, ya que ésta será siempre el objetivo ideal a realizar en la realidad. Tanto el prejuicio platónico, como el imperialismo de la excelencia son los factores clave a comprender en un análisis hermenéutico-crítico de la propuesta predominante en filosofía del deporte actualmente. Mientras que el prejuicio se refiere, más bien, al aspecto metodológico del internalismo, el imperialismo de la excelencia lo hace al contenido resultante de la aplicación de dicho método.

\section{El prejuicio platónico en la corriente internalista}

El objetivo metodológico principal del internalismo es, dada su orientación platónica, el de ofrecer una definición del deporte apelando a los caracteres determinantes de su forma, idea, o esencia. La ligazón entre el internalismo en filosofía del deporte y el platonismo desde una perspectiva metodológica no es algo nuevo. Esta ya ha sido sacada a la luz por D'Agostino, quien ha denominado y criticado al formalismo, la propuesta filosófica de Suits originaria del internalismo amplio, por ser platónico. Para D'Agostino,

los formalistas acaban abocados a un tipo de platonismo sobre el juego. Para ellos, los juegos son tipos ideales, que son sólo realizados de un modo imperfecto en sus supuestas ejemplificaciones. ${ }^{29}$

El formalismo toma como elemento definitorio del deporte las normas que crean los obstáculos innecesarios que permiten al participante alcanzar la excelencia física a través del juego, es decir, las normas constitutivas del juego. Toda propuesta internalista ha heredado esta tendencia de convertir la excelencia en el elemento definitorio del deporte, tal y como mostramos de modo claro en la nota 27. Dejando de lado el contenido de la propuesta internalista, es decir, su focalización en el concepto de excelencia, y centrándonos en su carácter más metodológico; la crítica de D’Agostino, así como la nuestra, afirman que la metodología del internalismo está basada en presupuestos analítico-platónicos que reducen la naturaleza del

28. Así, por ejemplo, para John S Russell, "las reglas deben ser interpretadas de modo que las excelencias necesarias para alcanzar la meta lúdica del juego no sean socavadas, sino mantenidas y potenciadas." Russell, "Are rules all an empire has to work with?" cit. p. 35. Igualmente, para Robert L. Simon: «Un deporte bien diseñado es aquel en el que los desafíos exigen habilidades complejas para resultar exitoso, el uso de diversas estrategias, y la necesidad para tomar decisiones en varias situaciones del juego. La mayor meta de la participación, entonces, es alcanzar la excelencia a través de superar el desafío». Simon, R. "Theories of sport" cit. 95.

29. D'Agostino, F.: "The ethos of games." p. 9 en Journal of the Philosophy of Sport Journal of the Philosophy of Sport 8(1), 1981, pp. 7-18. 
fenómeno deportivo apelando a una definición ideal del mismo que no es identificable ni realizable en la realidad.

Por ejemplo, en fútbol una norma constitutiva es no zancadillear a los rivales para impedirles conducir el balón. Según el formalista, desde el momento en que un futbolista zancadillea a los rivales, dejaría de practicar fútbol para hacer otra cosa. Si esto es así, entonces ninguna práctica deportiva real se puede ajustar a las exigencias normativas derivadas de la concepción ideal-normativa del deporte formalista, ya que en todos y cada uno de los deportes que conocemos, especialmente en los profesionales, los participantes utilizan la violación de las normas como estrategia, es decir, como parte del juego. De modo que, ningún deporte practicado en la realidad casa con la definición formalista del mismo. Una definición que no casa con la realidad difícilmente puede cumplir con su cometido de identificar los elementos definitorios que nos permiten separar la realidad en diversas categorías.

Basándonos en la crítica de D'Agostino al formalismo, afirmamos que éste constituye, también, como mostraremos más adelante, un punto débil en el resto de internalismos basados de modo consciente o inconsciente, en la propuesta metodológica internalista-platónica de Suits. Así pues, toda definición internalista del deporte será demasiado restrictiva, idealizada e incluso normativa. En el siguiente apartado criticamos el carácter reductivo e idealizado de la metodología internalista. Para ello, mostraremos las debilidades de la metodología platónica, entendida como prejuicio dominante en la filosofía del deporte en sus diversos niveles y órdenes de discurso, a través de un análisis hermenéutico. Más adelante, así como en posteriores trabajos, nos centraremos en la crítica referida al contenido resultante del prejuicio platónico, es decir, al imperialismo de la excelencia en la filosofía del deporte internalista.

\subsection{Lo reductivo de la metodología platónica}

Un primer punto flojo de la metodología platónica es su carácter reductivo de la realidad. En su búsqueda de definiciones que apelan a los caracteres determinantes de las cosas, la metodología analítico-platónica siempre acaba reduciendo la realidad de las mismas. Así, por ejemplo, siguiendo la definición formalista, que todas las prácticas deportivas estén constituidas esencialmente por normas, no implica que la realidad de la práctica deportiva se reduzca a la observación e imperio de éstas. Si sólo prestamos atención a este aspecto formalista, como bien muestra D'Agos-

Thémata. Revista de Filosofía N54 (2016) pp.: 125-148. 
tino, estamos dejando de lado otros elementos constitutivos de la realidad deportiva.

De hecho, la propia historia de la filosofía del deporte se desarrolla a partir de la complementación de las diversas concepciones del mismo por considerarlas demasiado reductivas o simples. ${ }^{30}$ Por ejemplo, como hemos afirmado, D'Agostino critica al formalismo no tener en cuenta las convenciones que nos llevan a aplicar las normas de un modo u otro. Igualmente, el internalismo amplio, a su vez, critica a D'Agostino no haber considerado el propósito último del juego, y al que las convenciones rinden tributo: la excelencia física. ${ }^{31}$ Toda nueva propuesta en filosofía del deporte ha criticado a sus predecesoras por haber ofrecido una definición demasiado reductiva y busca como principal objetivo definir el deporte de un modo más amplio.

No obstante, metodológicamente hablando, lo reductivo de las definiciones del deporte es consecuencia directa de la metodología empleada por todas y cada una de las propuestas internalistas. Así pues, todas pueden ser acusadas del mismo defecto: por mucho que incluyamos nuevos elementos en la definición de deporte, siempre se dejarán fuera otros aspectos que constituyen su realidad, y que más tarde podrán ser reclamados como definitorios del mismo por otras nuevas propuestas.

De otro modo, las propuestas internalistas del deporte obvian que el problema no está en el contenido que se otorga a la definición, sino en la intención metodológica misma de buscar una definición al modo platónico. Con ello, ignoran la paradoja que supone la propia búsqueda de definiciones: por mucho que se amplíe una definición, nunca se podrá captar la realidad completa del deporte. Esto es consecuencia de concebir la función de la filosofía del deporte siguiendo el prejuicio platónico heredado de la tradición iniciada por Suits.

Ninguna de las propuestas internalistas parece ser consciente de este prejuicio analítico-platónico. Para la hermenéutica, este es un error. Por ello, una hermenéutica del deporte, como afirmamos más arriba siguiendo a Gallagher, debe tener como su principal objetivo el de desvelar todos aquellos «factores epistemológicos, sociológicos, culturales, y lingüísticos» ${ }^{32}$ que influyen en nuestra comprensión del deporte. Sólo sacando a la luz estos factores podemos someterlos a crítica. Hemos, pues, de identificar nuestros prejuicios heredados acríticamente de la tradición con el fin de comprender las consecuencias del modo de ver la realidad que proyec-

30. LópezFrías, La filosofía del deporte actual cit.

31. Simon, R. L.: "Internalism and internal values in sport." en Journal of the Philosophy of Sport 27(1), 2000, pp. 1-16.

32. Gallagher, S. op. cit., p. 5.

Thémata. Revista de Filosofía $\mathrm{N}^{\circ} 54$ (2016) pp.: 125-148. 
tan. El internalismo deportivo no ha realizado hasta la fecha esta tarea, y ha seguido de modo inconsciente el prejuicio analítico-platónico.

No obstante, hemos de ir con precaución aquí. La hermenéutica no afirma que los prejuicios sean negativos en sí mismo y que hemos de revocarlos totalmente. Todo lo contrario, la reducción de la realidad a partir de preconcepciones o prejuicios en sí misma no es problemática para el hermeneuta, estos sirven como horizontes de sentido que proyectan un modo de interpretar la realidad. Los prejuicios son necesarios y constitutivos de nuestro modo de ser. No podemos funcionar sin prejuicios. ${ }^{33}$ La clave es ser conscientes de ellos. Cuando no lo somos, un prejuicio se vuelve ilegítimo, pues dirige nuestra actividad de un modo inconsciente y acrítico.

Volviendo a D'Agostino, aunque éste muestra acertadamente el carácter platónico del proceder internalista, no lleva esta crítica hasta el punto de poner en cuestión la totalidad del mismo, es decir, el prejuicio, sino que lo acepta acríticamente al igual que el formalismo al tratar de complementar la definición de deporte añadiendo la necesidad de recurrir a ciertas convenciones que muestran cómo deben aplicarse las normas para dar sentido al juego.

\subsection{La experiencia dulcificada del deporte del internalismo}

Puesto de manifiesto que la propuesta internalista hace uso inconsciente del prejuicio platónico-analítico que está en la base de su proceder, una de las principales consecuencias del mismo es que la definición del deporte nos cierra a otros modos de definirlo sesgando su realidad. Pero no sólo esto. Este modo de reducir la realidad del deporte a través del concepto internalista del mismo puede ser problemático. De hecho, como mostraremos en este apartado, la definición internalista de la práctica deportiva es problemática.

Así pues, la primera tarea de nuestra propuesta hermenéutica es la de poner de manifiesto que la definición del deporte como búsqueda de la excelencia es consecuencia de un prejuicio que, originado en la búsqueda platónica de una esencia del deporte, reduce la realidad del mismo. Más tarde, en un segundo momento, nos encargaremos del análisis crítico de dicho prejuicio. Con ello, insistimos, de nuevo, que la reducción de la realidad a través de una proyección de sentido determinada no es lo problemático en sí mismo, sino más bien el no darse cuenta de que dicha proyección no puede captar toda la realidad del deporte, considerando que la concepción del deporte como lucha por la excelencia tiene la última palabra. Esto

33. Gadamer, H. G. op. cit.

Thémata. Revista de Filosofía №54 (2016) pp.: 125-148. 
provoca un imperialismo de la excelencia que, en última instancia, es fruto del proceder metodológico creado por el prejuicio platónico-analítico dominante en el internalismo.

Como trataremos de demostrar, las propuestas internalistas llevan asociado en su proceder inductivo un ejercicio de verificación de su definición del deporte amparando ésta bajo un único sentido "bueno" del deporte. El propio Suits reconoce, en uno de los apéndices de The Grasshopper, que una de las limitaciones de su metodología platónica es que se puede caer en la paradoja de utilizar la reflexión deductiva para probar una definición que ya se tiene en mente de modo previo. Es decir, a propósito del internalismo de Suits, la metodología analítico-platónica que está a la base de su propuesta filosófica del deporte, pretendería probar la hipótesis inicial de que el juego consiste en la superación de obstáculos innecesarios creados por las normas en vez de conocer la realidad de juego en sí y que, por definición, es mucho más amplia y rica que su reducción a una definición del mismo.

Ser conscientes de que estamos dirigidos por un prejuicio, nos abre, como afirma Gadamer, a otros modos de comprender la realidad, así como a la posibilidad de analizar críticamente dicho prejuicio. Adoptando una postura hermenéutica, la experiencia, como mostrará Gadamer a modo de crítica hermenéutica al racionalismo heredero de Platón, no queda sesgada y dirigida acríticamente por una mentalidad reductiva que pretende comprender la realidad en función de unos elementos constitutivos universales:

cuando se piensa en la esencia de la experiencia sólo por referencia a la ciencia, como hace Aristóteles, se simplifica el proceso por el que se produce [...] no se lo puede describir simplemente como la formación, sin rupturas, de generalidades típicas. ${ }^{34}$

Cuando nos referimos a la reducción realizada por la tradición internalista del deporte a través de la verificación de su definición del mismo en clave de excelencia, consideramos que es posible aplicar la crítica realizada por Popper al proceso inductivo llevado a cabo por ciertas propuestas filosóficas como el psicoanálisis, el marxismo, y la filosofía nietzscheana. Según la cual, estas propuestas tratan de acomodar sus argumentos para verificar una hipótesis o idea general determinada. Por el contrario, Popper desarrolla la teoría de la "falsación". El objetivo de ésta no es verificar a través de la inducción, es decir, de las observaciones, sino que las obser-

34. Ibidem, p. 428.

Thémata. Revista de Filosofía No54 (2016) pp.: 125-148. 
vaciones se realicen con el fin de poner en jaque constante nuestra idea o hipótesis, es decir, falsarla:

la solución de un problema filosófico nunca es definitiva. No puede basarse en una prueba final ni en una refutación final: esto es una consecuencia de la irrefutabilidad de las teorías filosóficas. ${ }^{35}$

Tomando como analogía la crítica de Popper, afirmaremos que las propuestas internalistas del deporte se basan, al igual que el platonismo cuando trata de alcanzar dialécticamente la idea o forma de algo, en la verificación de dicha idea. Para el internalismo, una de sus principales pretensiones filosóficas pasa por tratar de alcanzar no sólo una definición universal del deporte sino, también, tratar de "desvelar" la verdad del mismo. Dicha objetividad o verdad viene determinada por la idea de excelencia que, como ocurre con el caso de las filosofías de la sospecha, tratan de acomodar el conjunto de datos de la experiencia para, de ese modo, hacerlas coincidir con una hipótesis central preestablecida que trata de ser verificada. En el caso de la propuesta internalista, el hecho de tratar de verificar la hipótesis dominante -la excelencia como verdad del deporte-, conlleva el ajuste forzoso de otros tipos de experiencia que, en otro tipo de condiciones epistemológicas, falsarían la naturaleza del deporte concebido esencialmente como lucha por la excelencia.

De hecho, podemos encontrar muchos ejemplos de elementos opuestos a la idea de excelencia que se dulcifican o acomodan para que todo quepa bajo dicha idea de verdad del deporte. Este es el ejemplo de la lucha o la tensión. En los trabajos internalistas vemos cómo los filósofos del deporte, tras observar que un componente definitorio del mismo es la lucha por vencer al otro, afirman que en el deporte hay una "dulce tensión" ${ }^{36}$ que da emoción al mismo convirtiéndolo, a la vez, en una lucha "civilizada" por la excelencia. De ese modo, el internalismo cree salvar aquellos fenómenos contrarios a su idea preconcebida de la excelencia. En cualquier caso, esto no deja de ser un ejemplo del proceder platónico-analítico propio del internalismo que, tal y como acabamos de ver, acomoda la idea de lucha contra

35. Popper, K.: "Metafísica y criticabilidad", p. 234 en David Miller (comp.), Popper. Escritos selectos, México: Fondo de Cultura Económica, 1995, pp. 225-234.

36. Kretchmar, R.S. "From test to contest" cit.; McLaughlin, D. W. y Torres, C. "Sweet tension and its phenomenological description".

Thémata. Revista de Filosofía №54 (2016) pp.: 125-148. 
el otro dentro de los límites establecidos por la noción de deporte como búsqueda de la excelencia.

Igualmente, Warren P. Fraleigh ${ }^{37}$ en línea con este modo dulcificado de concebir el deporte, afirma que el oponente no debe ser identificado como un obstáculo o un problema que hemos de superar a toda costa, sino como un facilitador. Con su oposición, el contrincante nos brinda la oportunidad a dar más de nosotros mismo y, al mismo tiempo, nos ayuda a superar nuestros límites con el fin de alcanzar la excelencia.

Tal concepto de competición concibe a los oponentes como la condición recíprocamente necesaria para la expresión y el desarrollo. La competición es vista [...] como una actividad con otro, en vez de contra él [...] Lograr la excelencia queda disponible, en principio, a todos los participantes incluso cuando no siempre se dan competiciones excelentes, bien jugadas. Dado que dicha excelencia es producida por todos los participantes, el sentido de la victoria refleja la reciprocidad de los oponentes como facilitadores. ${ }^{38}$

Dentro del orden de discurso en el que nos situamos en éste punto de nuestra argumentación, la importancia de nuestra propuesta hermenéutica trata de identificar y asumir aquellos límites de la experiencia del deporte que, por alguna razón, habían sido ignorados, olvidados o simplemente acomodados a un proceso encaminado a la verificación de la excelencia. La experiencia hermenéutica entendida como apertura, tal y como mostramos en el apartado anterior, abandona todo esfuerzo por acomodar inductivamente los datos de la experiencia deportiva con el fin de hacerlos coincidir con los límites establecidos por la noción del deporte como lucha por la excelencia. Desde esta expansión dentro de los límites de sentido hermenéutico, es posible identificar y atender al conjunto de experiencias y datos que, de otro modo, son obviados por la tradición internalista del deporte. Dichas experiencias propias de la facticidad deportiva interpretadas más allá de su limitación dialéctico-platónica, nos encaminan hacia otros modos de comprender el deporte en su conjunto como un todo abierto al mundo y a la experiencia sensible, corporal y emocional del hombre.

Adoptar este carácter abierto dialógico no implica eliminar o derribar todos nuestros prejuicios para alcanzar una mirada perfecta o pura de la realidad. Todo lo contrario, los prejuicios son necesarios y fructíferos. Por ejemplo, la idea del deporte como excelencia proporciona un ideal crítico muy valioso para evaluar la situación del deporte de élite actual. Para ser críticos, pues, es necesario ser consciente de nuestros propios prejuicios. Esto es lo que pretende nuestra propuesta hermenéutica: hacernos

37. Fraleigh, W. P.:Right actions in sport: Ethics for contestants, Champaign, IL: Human Kinetics Publishers, 1984.

38. Ibídem, pp. 84-85.

Thémata. Revista de Filosofía $\mathrm{N}^{\circ} 54$ (2016) pp.: 125-148. 
conscientes de los prejuicios que guían nuestra pre-comprensión del deporte para ver qué parte de la realidad muestran y cuál oculta. De esto modo, por un lado, aceptamos que somos siempre parte de una tradición, que estamos situados en un momento concreto y que, por lo tanto, esto conlleva la aceptación de ciertos prejuicios. Este es, como reclama Heidegger, el punto de partida de cualquier análisis hermenéutico tal y como afirmamos al principio de este trabajo:

A diferencia de lo que ocurre en las ciencias, el rigor del pensar no consiste só1o en la exactitud artificial —es decir, teórico-técnica- de los conceptos. Consiste en que el decir permanece puro en el elemento de la verdad del ser y deja que reine lo simple de sus múltiples dimensiones. Pero, por otro lado, lo escrito nos aporta el saludable imperativo de una reducción lingüística meditada y cuidada [...] corriente de la vida y que el pensar del ser desfigura la existencial [...] Lo que ocurre es que la dificultad no consiste en tener que encontrar un sentido especialmente profundo o en tener que construir conceptos intrincados, sino que se esconde en ese paso atrás que introduce al pensar en un preguntar que es capaz de experimentar, renunciando al opinar habitual de la filosofía. ${ }^{39}$

\subsection{La paradoja del tercer hombre en el internalismo}

Siguiendo con nuestra crítica a la metodología platónica del internalismo, y tratando en la medida de los posible de cubrir los diferentes órdenes de discurso en el que el prejuicio platónico atraviesa la propuesta internalista del deporte, recurriremos al estudio de la paradoja del tercer hombre platónica que, a nuestro parecer, puede ser perfectamente aplicada a la filosofía del deporte actual:

-Pienso que tú crees que cada Forma es una por una razón como ésta: cuando muchas cosas te parecen grandes, te parece tal vez, al mirarlas a todas, que hay un cierto carácter que es uno y el mismo en todas; y es eso lo que te lleva a considerar que lo grande es uno.

-Dices verdad, afirmó.

-¿Y qué ocurre con lo grande en sí y todas las cosas grandes? Si con tu alma las miras a todas del mismo modo, ¿no aparecerá, a su vez, un nuevo grande, en virtud del cual todos ellos necesariamente aparecen grandes?

-Tal parece.

-En consecuencia, aparecerá otra Forma de grandeza, surgida junto a la grandeza en sí y a las cosas que participan de ella. Y todos éstos, a su vez, otra Forma, en virtud

39. Heidegger, M.: Carta sobre el humanismo, Madrid: Alianza, 2000, pp. 14, 28 y 58.

Thémata. Revista de Filosofía $\mathrm{N}^{\circ} 54$ (2016) pp.: 125-148. 
de la cual todos ellos serán grandes. Y así, cada una de las Formas ya no será una unidad, sino pluralidad ilimitada. ${ }^{40}$

La clave del argumento del tercer hombre es que cuando generamos una idea de algo, siempre podemos compararla con una idea mayor de la que participa. Aplicando dicho argumento al caso de la metodología internalista, que como afirmamos se basa en la noción de excelencia, ésta siempre puede ser comparada con otra de grado mayor de la que participa. Toda idea de excelencia tiene la capacidad de autogenerar otra idea de excelencia todavía mayor, y, así, hasta el infinito. Esto es lo que Vlastos ${ }^{41}$ ha denominado como autopredicación de la idea. Esta crítica, queda más clara en una segunda versión del argumento del tercer hombre, en la que Platón afirma:

En consecuencia, no es posible que algo sea semejante a la Forma ni que la Forma sea semejante a otra cosa; porque, en tal caso, junto a la Forma aparecerá siempre otra Forma, y si aquélla fuese semejante a algo, aparecerá a su vez otra Forma, y jamás dejará de surgir otra Forma siempre nueva, si la Forma se vuelve semejante a lo que de ella participa. ${ }^{42}$

Esta segunda formulación de la paradoja platónica del tercer hombre se sitúa en otro nivel de discurso. Si bien la primera se refería más bien a un ámbito lógico, esta segunda se refiere a los elementos que se van agregando a la definición, los cuales pueden ampliarse de un modo infinito porque, en el caso que nos ocupa, la idea de excelencia siempre puede acabar participando de otras mayores con más elementos. En un terreno práctico, observamos cómo al deportista se le exige siempre más. Primero, que sea ejemplar para sus compañeros, luego para sus oponentes, luego como profesional, más adelante que sea ejemplo a seguir para los más jóvenes, y después, para los miembros de toda la sociedad. Y así sucesivamente. No parece haber pues, un límite claro a eso que sea la excelencia; siempre pueden generarse nuevas expectativas e ideas de excelencia mayor.

De hecho, en un plano teórico, esto es fácilmente detectable en la filosofía del deporte. Si bien la excelencia para el internalismo se refiere primordialmente al ámbito físico, es decir, al cultivo del cuerpo, ésta tiene un segundo tipo de ramificaciones más amplias. Para ser excelente deportivamente hablando hace falta dedicación, esfuerzo, conocerse a sí mismo, respetar al rival... Lo cual, en cualquier caso, va más allá y excede en mucho el ámbito de lo estrictamente físico. La prueba física (referida al

40. Platón, Parménides. 132a-b.

41. Vlastos, G.."The third man argument in the Parmenides." en The Philosophical Review, 63(3), 1954, pp. 319-349.

42. Platón, Parménides cit., 132e, 50.

Thémata. Revista de Filosofía $\mathrm{N}^{\circ} 54$ (2016) pp.: 125-148. 
controlar y lidiar con la finitud humana constitutiva de nuestra existencia) es, sin duda, un elemento clave del deporte, pero, para el internalista, éste no puede quedar reducido a ella ya que, a través de la superación de la prueba física, también ponemos a prueba otro tipo de habilidades, en especial, aquellas que tienen que ver con el ámbito de lo moral. Así, por ejemplo, Heather Reid denomina el deporte como una "actividad-perseguidora-de-conocimiento":

El movimiento físico voluntario era un producto y expresión de la mente/alma (psyche). El cuerpo atlético en forma, como producto de un movimiento voluntario e intencionado, es simplemente la muestra de la excelencia del alma. La competición atlética funcionaba dentro de la educación antigua no sólo como un entrenamiento físico, sino como un modo de cultivar almas fuertes y perseguidoras de la verdad. ${ }^{43}$

Aquí vemos cómo Heather Reid, en un principio define el deporte como lucha por la excelencia física pero, más adelante, afirma que esta excelencia participa de otra mayor, a saber, la del hombre con carácter disciplinado. A su vez, este ser disciplinado requiere de una excelencia mayor y así sucesivamente hasta alcanzar lo que se denomina como excelencia del carácter en general o del alma. El deporte así entendido es un modo de llevar a cabo la máxima socrática de "conocerse a sí mismo" inscrito en el Oráculo de Delfos. Es decir, tal y como afirma Simon, el deporte ya no es sólo una lucha por la excelencia sino, sobre todo, una actividad pedagógica. ${ }^{44}$ Siguiendo esta idea, Torres y McLaughlin afirman que:

participar seriamente en el deporte es preocuparse profundamente sobre los valores de la disciplina, así como, de un modo más general, del desarrollo humano y la excelencia. ${ }^{45}$

Volviendo a la paradoja del tercer hombre, creemos que podría ser una de las razones que llevó a Wittgenstein a formular su posición anti-definicionalista a través del concepto "aires de familia". Como muestra Wittgenstein en la cita al principio de este trabajo referente al concepto de juego, no puede encontrarse una idea global que abarque toda la realidad de aquello que denominamos como juego porque, de algún modo, siempre podemos aportar una idea más amplia del mismo.

43. Reid, H.: "Sport, philosophy and the quest for knowledge", p. 45 en Journal of the Philosophy of Sport 36, 2009, pp. 40-49.

44. Simon, R. "Theories of sport" cit.

45. McLaughlin D. y Torres C.: «More than games: Olympism as a moral approach to sport» en H. Reid y M. Austin, The Olympics and Philosophy, Lexington: University Press of Kentucky, 2012. 


\subsection{Del es al debe. Una idea demasiado demandante o nor- mativa del deporte}

Según las críticas anteriores, por su modo de proceder, el internalismo no sólo no reconoce el prejuicio platónico en el que está basado, sino que reduce la experiencia y definición del deporte a la búsqueda de la excelencia hasta un punto que dulcifica la realidad del mismo para acomodarla a sus cánones de la verdad. De ese modo, el internalismo genera un "imperialismo de la excelencia" a la hora de establecer los límites de la práctica deportiva.

Siguiendo con nuestro modo de proceder analizando hermenéuticamente los diferentes niveles de discurso propios de la propuesta internalista, consideramos que existe un último problema que deber ser denunciado. Dicho problema consiste en que la metodología platónico-analítica de la propuesta internalista no sólo reduce la experiencia del deporte y su definición, sino que convierte a esta última en un ideal normativo a alcanzar, en vez de en una descripción de la cosa a estudiar. Siendo esto así, podemos decir que los autores internalistas realizan un salto de lo descriptivo a lo normativo tal y como hemos visto en las citas de Reid y Simon más arriba. En éstas, de la definición descriptiva del deporte como búsqueda de la excelencia física se salta inductiva y espontáneamente a la concepción del mismo como una práctica pedagógica con exigencias normativas. Esto constituye, a nuestro juicio, un error.

Este salto de lo descriptivo a lo normativo se encuentra en la filosofía del deporte desde muy temprano, tanto como para localizarlo en Suits, considerado por muchos como su fundador. Concebido como padre del formalismo en la filosofía del deporte, es decir, de aquella corriente que define el deporte apelando a sus normas constitutivas, Suits establece una muy delgada línea entre lo descriptivo y lo normativo al formular su tesis de la incompatibilidad lógica y su noción de la actitud lúdica del participante.

La primera afirma que si el deporte se define por ser un conjunto de normas que generan obstáculos innecesarios con el fin de pasarlo bien, quebrar las normas es algo incorrecto en tanto que ello da por concluida la actividad lúdica. De este modo, hacer trampas y participar son incompatibles. Para participar en el deporte, pues, se deben seguir las normas en todo momento. Además, afirma Suits, que es necesario que los participantes entren en la práctica con una actitud lúdica, dispuestos a no buscar los medios más eficientes para alcanzar el fin del juego, sino aceptando los retos innecesarios planteados por la prueba. De este modo, la integridad

Thémata. Revista de Filosofía N54 (2016) pp.: 125-148. 
de las reglas y el espacio creado por ellas se mantienen intactos, siendo posible la práctica del deporte:

Jugar a un juego es formar parte de una actividad dirigida a lograr un estado de cosas determinado, usando sólo los medios que están permitidos por reglas específicas, en la que los medios permitidos por las reglas son más limitados en alcance de lo que serían en ausencia de las reglas, y en la que la razón para la sola aceptación de tales limitaciones es hacer posible tal actividad [...] las reglas en el juego: parecen entablar una relación peculiar con los fines [del juego.] Las reglas, pues, parece ser en cierto sentido inseparables de los fines. Romper una regla es convertir en imposible el [juego. De modo que uno no puede a la vez jugar y hacer trampas, sino que debe aceptar las normas para que el juego sea posible.]. ${ }^{46}$

Tanto la tesis de la no compatibilidad del jugar y hacer trampas, como la necesidad de adoptar una actitud lúdica para jugar son afirmaciones normativas derivadas de la constatación de que el deporte es definido esencialmente por sus normas. De este modo, de la descripción de la naturaleza del juego se salta directamente a lo normativo. Nosotros defendemos que este salto de lo descriptivo a lo normativo es una herencia del platonismo, que, a nuestro parecer, está a la base de la propuesta internalista en filosofía del deporte y que actúa como paradigma dominante, restrictivo y constriñe los límites de la experiencia y realidad del deporte.

Aunque el principal objetivo de Suits es el de definir el concepto de juego, es decir, la dilucidación de la Idea o Forma del deporte en sí. El platonismo a la base de su propuesta genera consecuencias normativas. Cuando postulamos un modelo ideal del deporte, como D'Agostino critica al formalismo, creamos un modelo perfecto del mismo al que debemos de aproximarnos pero nunca podremos realizar de modo pleno. Esto conlleva obligaciones morales referidas a lo que las cosas deben ser. De lo descriptivo se salta, así, a lo normativo. Lo cual, como bien mostró Kant, es problemático. ${ }^{47}$ A nuestro juicio, los polos normativo y descriptivo no deben confundirse. Realizar un salto injustificado, espontáneo e inductivo del uno al otro es erróneo, ya que describir la naturaleza del deporte es algo muy distinto a la tarea de prescribir cómo debe ser el deporte teniendo en

46. Suits, B.: "Whatis a game?" en Philosophy of Science, 34 (2), 1967, 148-156 y 150-151.

47. Así, por ejemplo, Kant muestra la ilegitimidad de este salto de lo descriptivo a lo normativo en su crítica a la filosofía moral griega, que es concebida como naturalizada o heterónoma. "Los principios empíricos no sirven nunca para fundamento de las leyes morales [...] estos principios no establecen más que heteronomía de la voluntad como fundamento primero de la moralidad, y precisamente por eso han de fallar necesariamente su fin [...] Pues la universalidad con que deben valer [las leyes morales] para todos los seres racionales sin distinción, la necesidad práctica incondicionada que por ello le es atribuida, desaparece cuando el fundamento de ella se deriva de la peculiar constitución de la naturaleza humana o de las circunstancias contingentes en que se coloca" Kant, I Fundamentación de la metafísica de las costumbres, Madrid: Tecnos, 1989, p. 80 y 82.

Thémata. Revista de Filosofía $\mathrm{N}^{\circ} 54$ (2016) pp.: 125-148. 
cuenta el conjunto de valores morales que se consideran como correctos en nuestras sociedades.

\section{Conclusión. El camino hacia una inversión del internalismo}

En este trabajo, hemos realizado el primer paso para llevar a cabo una hermenéutica crítica y práctica del deporte, a saber: la identificación de los prejuicios que dirigen la concepción dominante del deporte aplicando, para tal propósito, la metodología hermenéutica en los diferentes niveles y órdenes de discurso del internalismo. Al llevar a cabo esta tarea, hemos identificado un prejuicio platónico-analítico ligado a la búsqueda de una definición del deporte y heredado de Suits, padre del internalismo. Una vez sacado a la luz este modo de proceder, hemos puesto en cuestión cuatro consecuencias problemáticas de su puesta en práctica: (a) que la metodología platónico-analítica sesga la realidad sin que lo percibamos; (b) que ese sesgo que realiza de la realidad del deporte acaba dulcificándolo la realidad del deporte al apelar únicamente a la idea de excelencia; (c) que la intención de definir el deporte en base a la idea de excelencia es, cuando menos, problemática, en tanto que siempre se puede encontrar una idea mayor de excelencia. En cierto sentido, corremos el riesgo de llevar la definición del deporte como excelencia a un círculo vicioso; y (d) que el uso de la idea de excelencia es en sí equivocada porque nos hace dar un salto cuanto menos dudoso e injustificado de lo descriptivo a lo normativo generando, tal y como hemos visto, implicaciones normativas a raíz de la descripción de los caracteres definitorios del deporte. Con todo esto, hemos identificado cuáles son los límites de nuestra concepción dominante del deporte. En otro futuro trabajo procederemos a realizar una inversión de este platonismo en la filosofía internalista del deporte con el fin de mostrar de modo más claro que los límites de la idea de deporte son más amplios o, en palabras de Nietzsche, transvalorables.

\section{Referencias bibliográficas}

Aggerholm, K.: "Talent development, existential philosophy and sport: on becoming an elite athlete." New York: Routledge, 2015.

Conill-Sancho, J. (2012). "La superación del naturalismo en Ortega y Gasset." Isegoria 46: 167-192.

D'Agostino, F. (1981). "The Ethos of Games." Journal of the Philosophy of Sport_8(1): 7-18.

Edgar, A. (2014). Sport and art : an essay in the hermeneutics of

Thémata. Revista de Filosofía N54 (2016) pp.: 125-148. 
sport. London; New York, Routledge.

Fraleigh, W. P. (1984). Right actions in sport: Ethics for contestants, Human Kinetics Publishers Champaign, IL.

Gadamer, H.-G. (1977). Verdad y método : fundamentos de una hermenéutica filosófica, Salamanca, Ediciones Sígueme.

Gallagher, S. (1992). Hermeneutics and education. Albany, State University of New York Press.

Kretchmar, R. S. (1975). "From Test to Contest: An Analysis of Two Kinds of Counterpoint in Sport." Journal of the Philosophy of Sport 2(1): 23-30.

Kretchmar, R. S. (2007). "Dualisms, Dichotomies and Dead Ends: Limitations of Analytic Thinking about Sport." Sport, Ethics and Philosophy 1(3): 266-280.

Kretchmar, R. S. (2014). The philosophy of sport and analytic philosophy. Bloomsbury companion to the philosophy of sport. C. R. Torres. London, Bloomsbury: 41-52.

López Frías, F. J. (2014). La filosofía del deporte actual. Paradigmas y corrientes. Roma, (Qua.Pe.G) Università degli Studi di Roma "Foro It.

López Frías, F. J. (2016). Broad internalism and interpretation. The plurality of interpretivist approaches. Defining sport. Contemporary explorations. S. Klein. Lexington Books. En Prensa.

MacIntyre, A. (2001). Tras la virtud. Barcelona, Crítica.

Marmor, A. (2009). Social conventions: from language to law. Princeton, N.J, Princeton University Press.

Martinkova, I. and S. J. Parry (2012). Phenomenological approaches to sport. New York; London, Routledge.

McLaughlin, D. W. and C. R. Torres (2011). "Sweet Tension and its Phenomenological Description: Sport, Intersubjectivity and Horizon." Sport, Ethics and Philosophy Sport, Ethics and Philosophy 5(3): 270-284.

McNamee, M. (2007). "Sport, ethics and philosophy; context, history, prospects." Sport, Ethics and Philosophy 1(1): 1-6.

McNamee, M. J. and W. J. Morgan (2015). "Routledge Handbook of the Philosophy of Sport."

Moe, V. F. (2014). The philosophy of sport and continental philosophy. Bloomsbury companion to the philosophy of sport. C. R. Torres. London, Bloomsbury: 52-66;

Morgan, W. J. (2012). "Broad internalism, deep conventions, moral entrepreneurs, and sport." Journal of the Philosophy of Sport 39(1): 65-100. EDAF.

Nietzsche, F. and J. Mardomingo (2002). La gaya ciencia. Madrid,

Platón (2014). República ; Parménides ; Teeteto. Madrid, Gredos. 
Rawls, J. (1995). Liberalismo político. México, Fondo de Cultura Económica.

Rorty, R. (1979). Philosophy and the mirror of nature. Princeton, Princeton University Press.

Russell, J. S. (1999). "Are Rules All an Umpire Has to Work With?" Journal of the Philosophy of Sport 26(1): 27-49.

Russell, J. S. (2004). "Moral Realism in Sport." Journal of the Philosophy of Sport 31(2): 142-160.

Simon, R. L. (2000). "Internalism and Internal Values in Sport." Journal of the Philosophy of Sport 27(1): 1-16.

Simon, R. L. (2014). Theories of Sport. The Bloomsbury companion to the philosophy of sport en C. R. Torres. London, Bloomsbury Publishing: 83-98.

Standal, Ø. F. and V. F. Moe (2011). "Merleau-ponty Meets Kretchmar: Sweet Tensions of Embodied Learning." Sport, Ethics and Philosophy 5(3): 256-269.

Suits, B. (2005). The grasshopper: games, life and utopia. Peterborough, Ont, Broadview Press.

Torres, C. R. (2012). "Furthering Interpretivism's Integrity: Bringing Together Ethics and Aesthetics." Journal of the Philosophy of Sport 39(2): 299-319.

Torres, C. R. M., W (2014). "Olympism and the Olympic program: an intersubjective moral approach to sport." International journal of applied philosophy 28(2): 353-372.

Vlastos, G. (1954). "The Third Man Argument in the Parmenides." The Philosophical Review 63(3): 319-349.

Wittgenstein, L. (1988). Investigaciones filosóficas. Barcelona, Crítica. 
